Ambiente \& Água - An Interdisciplinary Journal of Applied Science
ISSN 1980-993X - doi:10.4136/1980-993X
www.ambi-agua.net
E-mail: ambi.agua@gmail.com

\title{
Detecção de cistos de Giardia spp. e oocistos de Cryptosporidium spp. na água bruta das estações de tratamento no município de Blumenau, SC, Brasil
}

\author{
doi:10.4136/ambi-agua.1853
}

Received: 02 Feb. 2016; Accepted: 08 Apr. 2016

\author{
Suelen Cristina Grott ${ }^{1 *}$; Brunna Hartmann ${ }^{2}$ Hercílio Higino da Silva Filho $^{1}$; \\ Regina Maura Bueno Franco ${ }^{3}$; Juliane Araújo Greinert Goulart ${ }^{1}$

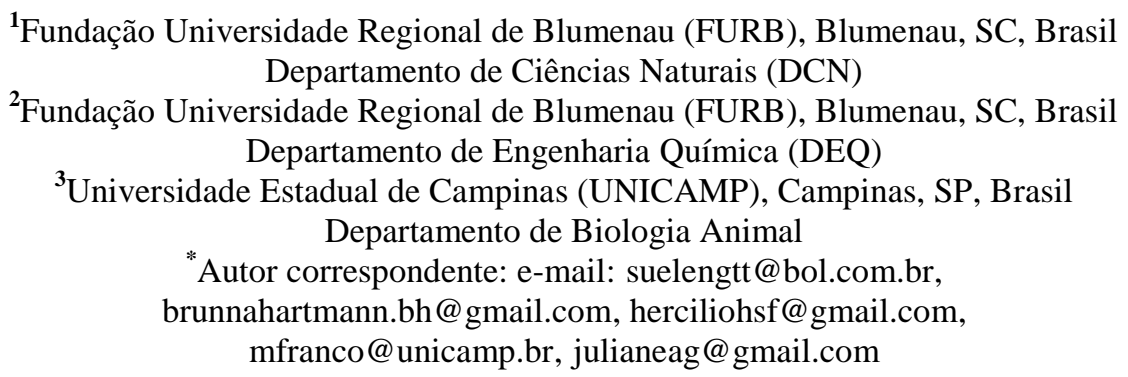

\section{RESUMO}

Giardia spp. e Cryptosporidium spp. são protozoários patogênicos de transmissão fecaloral de veiculação hídrica, que causam vários problemas de saúde, como doenças gastrointestinais associados com consumo de água contaminada. Devido à escassez de dados sobre a ocorrência destes protozoários em águas superficiais no Sul do Brasil, este estudo teve como objetivo analisar a presença de cistos e oocistos na água bruta das estações de tratamento de água no município de Blumenau, SC, Brasil. Para a pesquisa dos protozoários foi utilizada a metodologia de filtração em membranas de ésteres mistos de celulose, seguida por reação de imunofluorescência utilizando o kit Merifluor ${ }^{\circledR}$. Para as análises microbiológicas, empregou-se a metodologia do "Standard Methods for the Examination of Water and Wastewater"; para as análises físico-químicas foi utilizada sonda multiparâmetros. Cistos de Giardia spp. foram encontrados em 23,19\% das amostras e oocistos de Cryptosporidium spp. em 7,24\% do total de amostras analisadas $(\mathrm{n}=67)$. Todas as amostras analisadas apresentaram contaminação por Escherichia coli e 11,76\% apresentaram valores de turbidez da água bruta acima do recomendado. Nas análises da turbidez da água tratada 23,52\% apresentaram valores acima do preconizado pela Portaria 2914/2011. A detecção de protozoários patogênicos na água bruta das estações de tratamento de água aponta para a importância de adoção de medidas preventivas, como a proteção de áreas de mananciais e tratamento adequado do esgoto doméstico objetivando reduzir os riscos de transmissão de protozoários por meio da água de consumo humano na região de Blumenau, SC, Brasil.

Palavras-chave: água superficial, doenças de veiculação hídrica, protozoários. 


\title{
Detection of Giardia spp. cysts and Cryptosporidium spp. oocysts in untreated water collected from treatment plants in Blumenau, Santa Catarina State, Brazil.
}

\begin{abstract}
Giardia spp. and Cryptosporidium spp. are water-borne pathogenic protozoans spread through fecal-oral transmission which cause several health problems, to include gastrointestinal diseases associated with the consumption of contaminated water. There are limited data regarding the occurrence of these protozoans in surface waters in southern Brazil. The aim of this study was therefore to investigate the presence of cysts and oocysts in untreated water collected from water treatment plants in Blumenau, Santa Catarina State, Brazil. The methodology employed to study the protozoans was filtration through mixed cellulose ester membranes followed by the immunofluorescence reaction using the Merifluor ${ }^{\circledR}$ kit. Microbiological analysis was conducted using the Standard Methods for the Examination of Water and Wastewater and multi-parameter probes were used for the physico-chemical analysis. Cysts of Giardia spp. were found in 23.19\% of the samples and oocysts of Cryptosporidium spp. in $7.24 \%$ of the samples $(n=67)$. All of the samples analyzed were contaminated with Escherichia coli and $11.76 \%$ of the untreated water samples had turbidity values higher than the recommended limit. In the case of the treated water samples, $23.52 \%$ had turbidity values above the limit established by legislation (Portaria MS 2914/2011). The detection of pathogenic protozoans in the untreated water at the treatment plants highlights the importance of adopting preventative measures, such as the protection of areas where water is present and adequate treatment of domestic sewage, in order to reducing the risk of protozoan transmission via potable water.
\end{abstract}

Keywords: protozoans, surface water, water-borne diseases.

\section{INTRODUÇÃO}

A água desempenha um importante papel como veículo de transmissão de agentes biológicos (vírus, bactérias e parasitos), bem como uma fonte de contaminação por produtos químicos (efluentes industriais) (Nishi et al., 2012). Com isso a contaminação dos recursos hídricos por protozoários patogênicos de veiculação hídrica tem sido um fator de risco para problemas de saúde (Sato et al., 2013), especialmente em relação às gastroenterites.

Estima-se que apenas $18 \%$ da população mundial tem acesso à água potável de qualidade e, como consequência, mais de cinco milhões de pessoas morrem anualmente, devido as doenças associadas ao consumo da água contaminada e ao esgotamento sanitário inadequado (WHO, 2011). Cerca de $88 \%$ das mortes por diarreia em todo o mundo estão relacionadas a doenças de veiculação hídrica. Somente no Brasil, 396.048 pessoas foram internadas por diarreia em 2011; destas, 138.447 foram crianças menores de cinco anos (35\% do total) (Trata Brasil, 2013).

As doenças de veiculação hídrica, sobretudo aquelas causadas por protozoários intestinais, emergiram como um dos principais problemas de Saúde Pública nos últimos 25 anos (Karanis et al., 2007). Dentre os protozoários causadores de doenças de veiculação hídrica, Giardia e Cryptosporidium são os que apresentam as formas infectantes mais resistentes aos processos de desinfecção da água. Juntos foram responsáveis por cerca de 90\% dos surtos de gastroenterite ocorridos desde os anos 1980 em países como os Estados Unidos, Canadá e Japão (Karanis et al., 2007; Wang et al., 2011). Ambos protozoários se caracterizam por gerar nos pacientes acometidos quadros de diarreia de diversa severidade, 
principalmente em indivíduos imunocomprometidos (Lobo et al., 2009). Estima-se que $20 \%$ da população mundial esteja parasitada por Giardia spp. (Rockwell, 2003). No Brasil, a criptosporidiose e a giardiose representam importante causa de morbidade em crianças de 0 a 5 anos (Carvalho-Almeida et al., 2006, Gonçalves et al., 2006).

De acordo com Savioli et al. (2006), devido aos impactos no desenvolvimento socioeconômico, especialmente nos países em desenvolvimento, estes protozoários estão incluídos na lista de doenças negligenciadas em humanos pela Organização Mundial da Saúde.

A existência destes protozoários em reservatórios que são utilizados para tratamento e abastecimento público de água ocorre principalmente devido à contaminação do ambiente com as formas infectantes de Cryptosporidium spp. e Giardia spp. provindos do esgotamento doméstico e industrial (Kumar et al., 2016). Com isso o monitoramento dos protozoários patogênicos Giardia spp. e Cryptosporidium spp. em estações de tratamento de água tornou-se obrigatório no Brasil, segundo a Portaria no 2.914/2011 do Ministério da Saúde (Brasil, 2011). Para isso as companhias de tratamento de água devem realizar o monitoramento dos mananciais de acordo com a concentração de Escherichia coli (quando for identificada média geométrica anual maior ou igual a $1.000 \mathrm{E}$. coli/100 mL deve-se realizar o monitoramento dos protozoários Giardia spp. e Cryptosporidium spp.). Porém, para que esse monitoramento seja realizado, alguns problemas precisam ser solucionados, como a falta de profissionais treinados e altos custos para realização da metodologia de detecção destes protozoários.

No município de Blumenau, como em toda bacia do Rio Itajaí-Açu poucos foram os estudos realizados investigando a presença de Giardia spp. e Cryptosporidium spp. As publicações realizadas referem-se somente a creches (Andrade et al., 2008), e animais silvestres (Volotão et al., 2008).

No município de Blumenau até 2012, de todo esgoto produzido na cidade, apenas 4,8\% era tratado, o restante era lançado in natura nos corpos receptores da região (Trata Brasil, 2013). No ano de 2013, os serviços de redes coletoras e tratamento de esgotos chegaram a 30\% (Odebrecht, 2016). De acordo com o Plano Municipal de Saneamento do município de Blumenau, SC, Brasil, espera-se que até 2050 todo esgoto produzido seja devidamente tratado Blumenau, 2009).

Diante disso, os objetivos do presente estudo foram investigar a ocorrência de oocistos de Cryptosporidium spp. e cistos de Giardia spp. em amostras de água bruta destinadas ao abastecimento público no município de Blumenau; verificar a qualidade microbiológica e físico-química das mesmas e relacionar os parâmetros físico-químicos e indicadores bacteriológicos com a eventual presença desses protozoários.

\section{MATERIAIS E MÉTODOS}

\subsection{Local de estudo}

O município de Blumenau está inserido, na sua maior parte, na bacia hidrográfica do Rio Itajaí, região leste do Estado de Santa Catarina, Brasil (Figura 1). A bacia apresenta uma extensão de $15.000 \mathrm{~km}^{2}$ e se estende do planalto catarinense até o litoral, sendo o principal curso de água, dentro da rede hidrográfica do Estado de Santa Catarina, e a maior bacia hidrográfica da Vertente Atlântica. No município, o rio Itajaí-Açu recebe lançamentos de cinco ribeirões, sendo eles: ribeirão Garcia, ribeirão da Velha, ribeirão Itoupava, ribeirão do Testo e ribeirão Salto do Norte. O município possui uma população de 309.011 habitantes, distribuída em $518,497 \mathrm{~km}^{2}$ de área, densidade populacional de 594,44 hab./km², dos quais 95,5\% vivem em área urbana e 4,5\% em área rural (IBGE, 2010). 


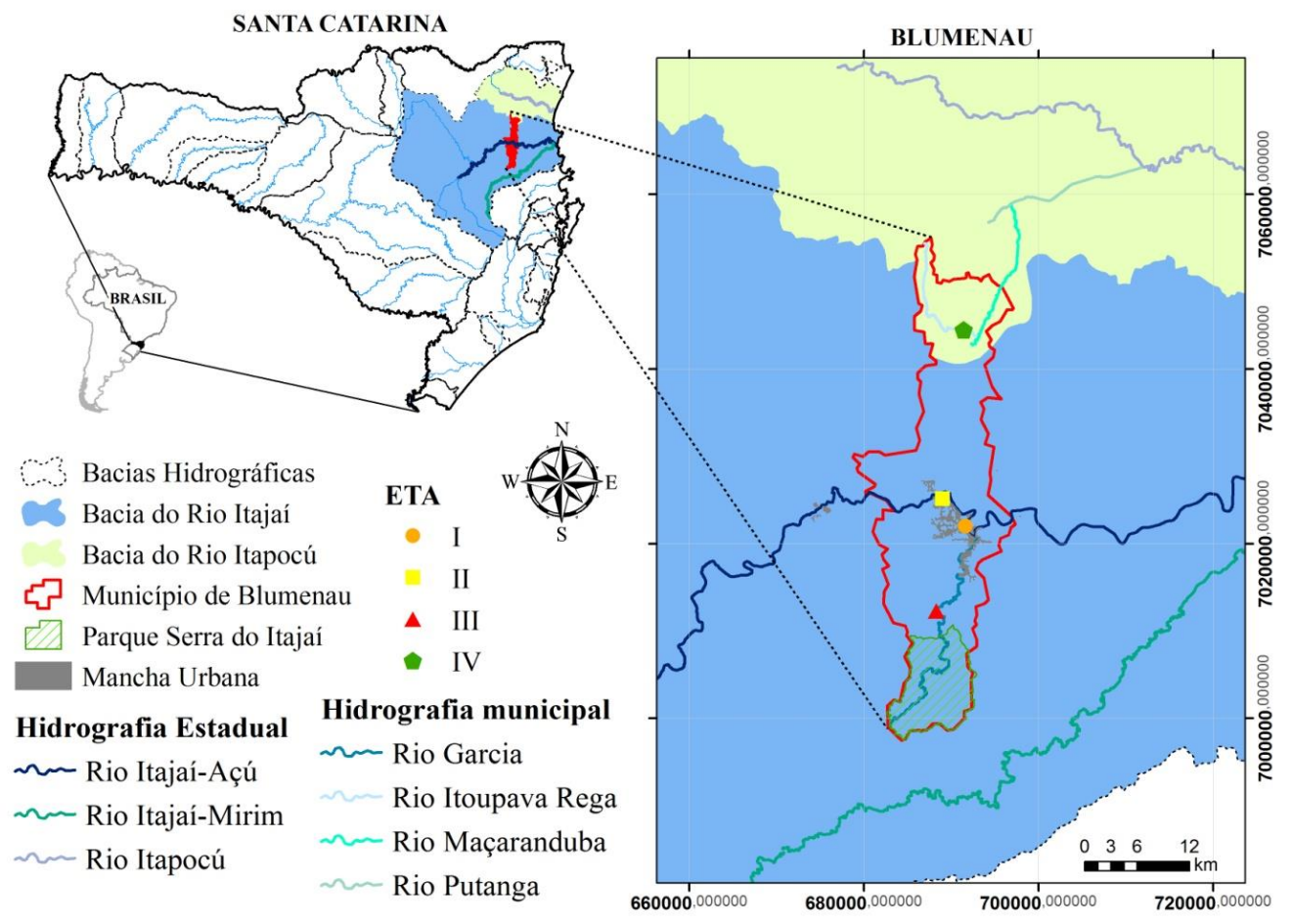

Figura 1. Localização da área de estudo e das estações de tratamento de água no município de Blumenau, Santa Catarina, Brasil.

O clima da região é classificado como subtropical úmido, para todas as estações, caracterizado por verões quentes e chuvosos, com umidade relativa do ar de $84,2 \%$. As estações do ano são bem definidas, com invernos mais secos (IBGE, 2010).

\subsection{Amostragem}

As coletas de água bruta foram realizadas mensalmente, entre os meses de agosto de 2013 a agosto de 2014, totalizando 14 coletas. No mês de outubro de 2013 foram realizadas duas coletas nas estações, porém, essa periodicidade não foi mantida devido ao curto período para análise do material.

As estações incluídas no presente estudo foram as quatro unidades de tratamento de água do Serviço Autônomo Municipal de Água e Esgoto (SAMAE) de Blumenau, indicadas neste trabalho como ETA I, II, III e IV (Figura 1).

A ETA II é a maior estação do município, captando água do Rio Itajaí-Açu e é responsável por $70 \%$ do abastecimento no município (SAMAE, 2013).

Além dos quatro pontos de coleta de água, também foram realizadas coletas no Parque Nacional da Serra do Itajaí (PNSI), com 57.374 hectares, por ser um ambiente preservado, com vegetação densa e nativa, sem a presença de habitações humanas e criações de animais. O PNSI localiza-se a $23 \mathrm{~km}$ da área central de Blumenau, SC, Brasil.

\subsection{Pesquisa de oocistos de Cryptosporidium spp. e cistos de Giardia spp.}

$\mathrm{Na}$ primeira etapa da pesquisa foi realizado o experimento de contaminação artificial de uma amostra de água provenientes da ETA II. Tal prática se faz necessário para verificar a taxa de recuperação e precisão da técnica.

Inicialmente foi realizada a contagem dos protozoários presentes em três alíquotas de $10 \mu l$ da suspensão "controle positivo" do kit Merifluor ${ }^{\circledR}$. Estas alíquotas foram examinadas por reação de imunofluorescência direta considerada a média do número de formas parasitárias detectadas em três poços da lâmina de imunofluorescência para cálculo da dose empregada para contaminação das amostras-controle. 
Para avaliar a recuperação do método, seis alíquotas de $2 \mathrm{~L}$ de água bruta proveniente da ETA II foram contaminadas com aproximadamente 1000 oocistos de Cryptosporidium e cistos de Giardia presente no kit Merifluor ${ }^{\circledR}$ e realizado todo o procedimento analítico. Uma alíquota de $2 \mathrm{~L}$ sem contaminação foi processada para detecção de cistos e oocistos pré-existentes na amostra.

$\mathrm{Na}$ segunda etapa as amostras foram colhidas de acordo com os procedimentos do "Method 1623: Cryptosporidium and Giardia in water by Filtration/IMS/FA" (USEPA, 2012) e armazenadas em galões plásticos previamente higienizados com solução de Tween 80 a $0,01 \%$. As amostras $(10 \mathrm{~L})$ foram concentradas pela técnica de filtração em membranas de ésteres mistos de celulose ( $47 \mathrm{~mm}$ de diâmetro e porosidade de 3,0 $\mu \mathrm{m}$ ) de acordo com Franco et al. (2001).

As amostras de água foram filtradas mediante bomba de vácuo (fluxo de $4 \mathrm{~L} / \mathrm{min}$.) e sistema porta-filtro; após filtração, o material foi eluído a partir da superfície da membrana mediante a lavagem e raspagem da mesma, com solução de Tween 80 a $0,01 \%$. O material resultante foi concentrado por dupla centrifugação (1050 x $g ; 10$ min.).

A detecção dos protozoários foi realizada por microscopia de imunofluorescência direta, conforme as orientações presentes no kit Merifluor ${ }^{\circledR}$ (Meridian Bioscience Diagnostics, Cincinnatti, EUA).

O cálculo do número médio de cistos e oocistos por litro (L) foi realizado de acordo com Equação 1 descrita por Cantusio Neto e Franco (2004):

$$
X=\frac{\text { Número de oocistos ou cistos visualizados }}{\text { Volume de sedimento analisado }(\mu \mathrm{L})} \times \frac{\text { Volume do sedimento obtido }(\mu \mathrm{L})}{\text { Volume filtrado da amostra }(\mathrm{L})}
$$

Durante o período de análise das amostras foram realizados controles negativos, para os quais foram filtrados 2 litros de água destilada após o processamento das amostras mensais, seguindo os mesmos procedimentos utilizados nas amostras coletadas em campo.

\subsection{Determinação dos parâmetros físico-químicos}

Os parâmetros físico-químicos analisados no dia da coleta foram realizados com o auxílio de uma sonda multiparâmetros (Hidrolab DS5X®) e incluíram os parâmetros: temperatura $\left(\mathrm{C}^{\mathrm{o}}\right)$, turbidez $(\mathrm{uT})$, potencial hidrogeniônico $(\mathrm{pH})$, oxigênio dissolvido (mg.L-1), sólidos totais (ST) e teor de nitrogênio.

Os dados relativos à turbidez da água tratada foram fornecidos pelos laboratórios do SAMAE.

\subsection{Análises microbiológicas e pluviométrica}

Os dados microbiológicos foram fornecidos pelo laboratório central do SAMAE. As análises foram realizadas utilizando a técnica de membrana filtrante e tubos múltiplos (coliformes totais e termo-tolerantes e Escherichia coli) e processadas durante testes de rotina pela companhia de água, de acordo com a metodologia descrita pelo "Standard Methods for the Examination of Water and Wastewater" (APHA, 2005).

Os dados pluviométricos foram obtidos junto ao Centro de Operação do Sistema de Alerta - CEOPS/FURB.

Os valores de turbidez da água bruta e $\mathrm{pH}$ foram analisados de acordo com a Resolução do CONAMA $n^{\circ} 357 / 2005$, a fim de verificar se os valores estavam de acordo com os índices estabelecidos pela legislação.

\subsection{Tratamento estatístico dos dados}

Os resultados do monitoramento dos protozoários patogênicos nas amostras de água das estações de tratamento de água foram analisados utilizando estatística descritiva: média 
aritmética, valores máximos e mínimos.

Estudos de correlação utilizando o teste paramétrico de Correlação de Pearson foram realizados para a verificação da associação entre a concentração de protozoários e indicadores físicos e químicos e bacteriológicos. Quando os dados incluíam "zeros", a média foi calculada utilizando $\log (\mathrm{y}+1)$ a fim de tornar os valores homogêneos.

Em todas as análises estatísticas realizadas, considerou-se como estatisticamente significantes valores de p $<0,05$. A média geométrica para o cálculo dos dados de E. coli foi realizada utilizando a seguinte Equação 2.

$$
\bar{x}_{g}=\sqrt[n]{\chi_{1} \cdot x_{2} \cdot \cdots \cdot x_{n}}
$$

em que:

$\mathrm{X} 1, \mathrm{X} 2 \ldots . . . \mathrm{Xn}$ são os valores de E. coli registrados nas análises da água bruta das estações de tratamento de água.

\section{RESULTADOS E DISCUSSÃO}

\subsection{Pesquisa de oocistos de Cryptosporidium spp. e cistos de Giardia spp.}

A metodologia de filtração em membranas seguida de reação de imunofluorescência direta apresentou valores de recuperação dentro do estabelecido para o protozoário Giardia spp.,(recuperação de 55,58\% e desvio padrão de 36,45\%) conforme recomendado pela Agência de Proteção Ambiental dos Estados Unidos da América (USEPA, 2012).

Para Cryptosporidium spp. a recuperação ficou abaixo do recomendado (recuperação de 29,3\% e desvio padrão de 39,57\%), possivelmente devido a alta turbidez da água do manancial (ETA II). As sujidades presentes na amostra concentrada pela técnica de filtração em membranas é um dos maiores problemas enfrentados quando da realização da etapa de recuperação de oocistos (Franco et al., 2012) e tal fato foi corroborado por Cantusio Neto et al. (2010) em estudo realizado em Campinas, SP.

No controle negativo dos testes de recuperação foram utilizados $2 \mathrm{~L}$ de água proveniente do manancial ETA II e não foram detectados cistos e oocistos.

Durante o estudo foram processadas 67 amostras de água bruta dos mananciais e realizados seis controles negativos e seis controles positivos. Das amostras analisadas, 23,19\% (n=16) apresentaram cistos de Giardia spp. e 7,24\% (n=5) oocistos de Cryptosporidium spp..

No PNSI, na ETA III e VI não foi detectada a presença dos protozoários patogênicos Giardia spp. e Cryptosporidium spp.. No PNSI esse resultado pode ser explicado pelo fato do mesmo possuir 99,06\% de área coberta por floresta nativa e encontrar-se protegido por lei.

A ETA III, que utiliza o Ribeirão Garcia como fonte de captação, recebe suas águas das nascentes do PNSI, localizadas próximas à estação e apresenta 97,19\% de áreas preservadas com floresta nativa. Da água proveniente do PNSI, estima-se que aproximadamente 500 mil pessoas são beneficiadas direta e indiretamente, tanto em Blumenau, como nos demais municípios de abrangência do PNSI (Brasil e Instituto Chico Mendes, 2009). A ETA III abastece atualmente nove dos 35 bairros do município.

A ETA IV capta sua água do Ribeirão Itoupava Rega. O local é caracterizado por morros e vales estreitos, com densa área de vegetação e a água produzida abastece o bairro em que está localizada - Vila Itoupava. A bacia da ETA IV conta com apenas $30,82 \mathrm{~km}^{2}$, sendo que destes $67,92 \%$ são áreas de floresta nativa e 32,07\% são de outros usos, principalmente pequenas propriedades rurais.

Quanto às amostras positivas, verificou-se que apenas duas estações, a ETA I e a ETA II apresentaram amostras de água bruta contaminadas com cistos e oocistos. A maior ocorrência 
foi registrada para ETA I com valores de "não detectável" a 454 cistos de Giardia spp. e valores de Cryptosporidium spp. de "não detectável" a 254 oocistos durante o período das análises. Tanto a ETA I como a ETA II utilizam água do mesmo manancial - Rio Itajaí-Açu (Tabela 1). Os valores apresentam-se semelhantes aos compilados por Bastos et al. (2004), que verificaram a ocorrência de elevadas concentrações de cistos e oocistos em diversos mananciais no Brasil. Sato et al. (2013) descrevem que num total de 206 amostras coletadas em 28 locais no Estado de São Paulo, Giardia spp. foi detectada em 49,5\% das amostras em concentrações de 0,1 cistos/L até 97 cistos/L, e Cryptosporidium spp. foi detectado em 9,2\% das amostras em concentrações entre 0,1 oocisto/L a 6 oocistos/L, pelo método de concentração por filtração em membranas.

O Rio Itajaí-Açu, utilizado na captação de água para o tratamento público das ETA I e II é utilizado na captação de água da maioria dos municípios localizados ao longo do seu curso (Itajaí, Navegantes, Ilhota, Gaspar, Indaial, Blumenau, Apiúna, Lontras, Rio do Sul e Taió). O município de Blumenau retira entre $70 \%$ e $75 \%$ da água potável consumida na cidade, por meio destas estações. Juntas, as estações abastecem $68,5 \%$ dos bairros do município.

Nos experimentos realizados com controles negativos não foi detectada a presença de cistos e oocistos. $\mathrm{O}$ resultado sugere que não houve contaminação durante todo o processo de filtração e eluição das membranas ou confecção das lâminas.

Tabela 1. Resultados relativos à ocorrência de oocistos de Cryptosporidium e cistos de Giardia na água bruta das ETAs I, II, III e IV e no PNSI no município de Blumenau, SC, Brasil.

\begin{tabular}{|c|c|c|c|c|c|c|}
\hline \multirow{2}{*}{ ETA } & & \multirow{2}{*}{$\begin{array}{c}\mathrm{N}^{\mathrm{o}} \text { de amostras } \\
\text { positivas/Total de } \\
\text { amostras analisadas }\end{array}$} & \multirow{2}{*}{$\begin{array}{l}\text { Frequência } \\
(\%)\end{array}$} & \multicolumn{3}{|c|}{ Número de organismos/L } \\
\hline & & & & Média & (Máx.- Mín.) & Desvio padrão \\
\hline $\mathrm{I}$ & & $8 / 14$ & 57,14 & 101,5 & $(454-36)$ & 115,87 \\
\hline II & $\dot{\hat{n}}$ & $8 / 14$ & 57,14 & 141,1 & $(77-40)$ & 142,06 \\
\hline III & $\stackrel{3}{3}$ & $\mathrm{ND} / 14$ & - & N/D & - & - \\
\hline IV & 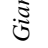 & $\mathrm{ND} / 14$ & - & N/D & - & - \\
\hline PNSI & & $\mathrm{ND} / 11$ & - & N/D & - & - \\
\hline I & $\begin{array}{l}\dot{2} \\
\stackrel{2}{\infty} \\
\equiv\end{array}$ & $3 / 14$ & 21,42 & 127,6 & $(254-77)$ & 108,97 \\
\hline II & $\equiv$ & $2 / 14$ & 14,28 & 286 & $(454-118)$ & 237,58 \\
\hline III & 苛 & $\mathrm{ND} / 14$ & - & N/D & - & - \\
\hline IV & $\hat{气}$ & $\mathrm{ND} / 14$ & - & N/D & - & - \\
\hline PNSI & $E$ & $\mathrm{ND} / 11$ & - & $\mathrm{N} / \mathrm{D}$ & - & - \\
\hline
\end{tabular}

Nota: ND: não detectado.

Cistos de Giardia spp. foram detectados em praticamente todos os meses, exceto nos meses de novembro de 2013 e agosto de 2014. Quanto à presença de oocistos de Cryptosporidium spp. os mesmos foram encontrados distribuídos ao longo de cinco meses, sendo quatro destes entre as estações com clima mais elevado (setembro, outubro de 2013 e janeiro e março de 2014) e somente uma ocorrência no inverno (julho/2014).

\subsection{Parâmetros físico-químicos}

Para os parâmetros físico-químicos, foram analisadas 67 amostras, distribuídas entre os cinco pontos de coleta, relativas à turbidez da água bruta que chega às estações de tratamento de água. Destas, oito $(11,76 \%)$ amostras apresentaram valores superiores a $100 \mathrm{uT}$ (valor máximo recomendado pela Resolução CONAMA 357/2005), sendo que em quatro (5,88\%) amostras os valores foram superiores a $500 \mathrm{uT}$, na ETA I e II (Tabela 2). Cabe ressaltar que os níveis de turbidez deste manancial (Rio Itajaí Açu) são normalmente mais elevados em relação aos demais mananciais. Isso ocorre principalmente devido às características do solo (Neossolos Flúvicos), possuindo cores mais escuras e texturas de diferentes grânulos. Além disso, o constante lançamento de efluentes domésticos e industriais no rio, bem como fezes de 
animais que ocupam áreas próximas à bacia hidrográfica, e são carreadas pela água da chuva, faz com que a turbidez mantenha-se acima da média dos demais pontos.

Não foi verificada correlação estatisticamente significante entre os dados de ocorrência de cistos de Giardia spp. e turbidez da água bruta ( $\mathrm{r}=-0,115 ; p=0,357 ; \mathrm{n}=67$ ). Para Cryptosporidium spp., foi verificada uma fraca correlação em relação aos valores de turbidez da água bruta ( $\mathrm{r}=0,280 ; p=0,022 ; \mathrm{n}=67$ ).

$\mathrm{Na}$ pesquisa foi verificada a presença de poucas amostras positivas mesmo com a detecção de altos valores para o parâmetro turbidez. Isso corrobora com os trabalhos realizados por Crockett (2004), que não obtiveram valores significativos de correlação em relação a este parâmetro. Cabe ressaltar também que a turbidez da água é o maior fator limitante da técnica de filtração em membranas, pois pode ocorrer rápida obstrução da malha filtrante com consequente redução do volume efetivamente filtrado e necessidade de substituição das membranas para filtrar todo o volume estipulado, o que pode ocasionar a perda de estruturas infectivas (Franco et al., 2012).

Quanto aos valores da turbidez da água tratada, verificou-se que 16 (23,52\%) das amostras analisadas, apresentaram valores acima de 0,5 uT (valor recomendado pela Portaria 2914/2011, para 95\% das amostras mensais, Brasil, 2011).

Dessas amostras, quatro $(7,27 \%)$ apresentaram valores acima de 1,0 uT, ficando acima do recomendado para os efluentes de filtração rápida, que estabelece que entre os $5 \%$ das amostras que apresentarem valores superiores a 0,5 uT, essas não poderão apresentar valores superiores a $1 \mathrm{uT}$. Essas amostras concentraram-se na ETA II, sempre após períodos com registro de elevados volumes de precipitação. Quando realizada correlação entre a precipitação e ocorrência dos protozoários na água bruta, não foi verificada correlação estatisticamente significativa entre os parâmetros analisados.

As amostras $(\mathrm{n}=5)$ que apresentaram concentrações de oocistos de Cryptosporidium spp. acima de 3,0 oocistos/L (Portaria 2914/2011, Art. 31, recomenda-se que o valor de turbidez seja menor ou igual a 0,3 uT em 95\% das amostras mensais ou uso de processo de desinfecção que comprovadamente alcance a mesma eficiência de remoção de oocistos de Cryptosporidium spp.) verificou-se que 3 amostras obtiveram valores superiores a $1 \mathrm{uT}$.

Quando analisada a correlação entre os valores de turbidez da água bruta em relação aos índices pluviométricos, verificou-se que estes apresentaram correlação fraca positiva $(\mathrm{r}=0,25 ; p=0,04 ; \mathrm{n}=67)$. A análise do $\mathrm{pH}$ indicou que todas as amostras se encontram dentro do estabelecido, ficando os valores entre 6,0 a 9,0 (CONAMA, 2005) e que não houve correlação entre $\mathrm{pH}$ e a ocorrência de protozoários patogênicos no manancial.

Tabela 2. Faixas de turbidez da água bruta e tratada, referenciando os valores recomendados pela Portaria 2914/2011 (Brasil, 2011), para remoção de oocistos e cistos de Cryptosporidium spp. e Giardia spp., no município de Blumenau, SC, Brasil.

\begin{tabular}{|c|c|c|c|c|c|c|c|}
\hline \multirow[b]{2}{*}{ ETA } & \multirow[b]{2}{*}{$\begin{array}{l}\mathrm{N}^{\circ} \text { amostras } \\
\text { analisadas }\end{array}$} & \multicolumn{3}{|c|}{ Turbidez água tratada } & \multicolumn{3}{|c|}{ Turbidez água bruta } \\
\hline & & $\begin{array}{c}\text { Acima de } 0,5^{*} \\
\text { uT }^{* * *}\end{array}$ & $\begin{array}{c}\text { Acima de } \\
1.0 \mathrm{uT}\end{array}$ & Máx./Mín. & $\begin{array}{l}\text { Acima de } \\
100 \mathrm{uT}^{* * * *}\end{array}$ & $\begin{array}{l}\text { Acima de } \\
500 \mathrm{uT}\end{array}$ & Máx./Mín. \\
\hline I & 13 & $2(15,38 \%)$ & - & $0,86 / 0,10$ & $2(15,38 \%)$ & $2(15,38 \%)$ & $1360 / 20,3$ \\
\hline II & 14 & $1(7,14 \%)$ & $2(14,28 \%)$ & $1,95 / 0,18$ & $2(14,28 \%)$ & $2(14,28 \%)$ & $934 / 18,3$ \\
\hline III & 14 & $5(35,74 \%)$ & - & $0,72 / 0,15$ & - & - & $14,8 / 1,21$ \\
\hline IV & 14 & $6(42,85 \%)$ & - & $0,97 / 0,18$ & - & - & $94,8 / 9,66$ \\
\hline
\end{tabular}

"Valores recomendados pela Portaria 2914/2011, para remoção de Cryptosporidium spp. e Giardia spp..

*** uT: refere-se à unidade de turbidez.

***: Valor recomendado pela resolução do CONAMA, 357/2005.

\subsection{Análises microbiológicas}

As amostras de água bruta apresentaram contaminação por bactérias do grupo coliforme,

Rev. Ambient. Água vol. 11 n. 3 Taubaté - July. / Sep. 2016 
em todas as amostras analisadas (Tabela 3).

De acordo com a Portaria 2914/2011, quando for identificada média geométrica anual maior ou igual a $1.000 \mathrm{E}$. coli/ $100 \mathrm{~mL}$ deve-se realizar monitoramento dos protozoários patogênicos no(s) ponto(s) de captação de água. Neste estudo os valores ficaram abaixo do exigido pela legislação para o monitoramento dos protozoários. No PNSI não foram realizadas análises bacteriológicas, pois esse ponto não é monitorado pelo SAMAE, que forneceu esses dados para a pesquisa.

Tabela 3. Qualidade bacteriológica da água bruta das estações de tratamento de água de Blumenau, SC, Brasil.

\begin{tabular}{|c|c|c|c|c|c|}
\hline \multirow{2}{*}{ ETA } & \multicolumn{2}{|c|}{ Coliformes totais (NMP/100 mL) } & \multicolumn{3}{|c|}{ Escherichia coli (NMP/100 mL) } \\
\hline & $\begin{array}{c}\mathrm{N}^{\mathrm{o}} \text { de amostras } \\
\text { analisadas }\end{array}$ & $\begin{array}{c}\text { Média } \\
\text { (Máx./ Mín.) }\end{array}$ & $\begin{array}{c}\mathrm{N}^{\mathrm{o}} \text { de amostras } \\
\text { analisadas }\end{array}$ & $\begin{array}{c}\text { Média } \\
\text { (Máx./ Mín.) }\end{array}$ & $\begin{array}{c}\text { Média } \\
\text { geométrica }\end{array}$ \\
\hline I & 26 & $\begin{array}{c}660,30 \\
(1600 / 240)\end{array}$ & 41 & $\begin{array}{c}124,82 \\
(430 / 23)\end{array}$ & 95,43 \\
\hline II & 10 & $\begin{array}{c}423,0 \\
(1600 / 240)\end{array}$ & 32 & $\begin{array}{c}84,93 \\
(240 / 33)\end{array}$ & 71,46 \\
\hline III & 48 & $\begin{array}{c}270,83 \\
(540 / 240)\end{array}$ & 48 & $\begin{array}{c}50,18 \\
(240 / 23)\end{array}$ & 39,29 \\
\hline IV & 35 & $\begin{array}{c}241,45 \\
(350 / 1,7)\end{array}$ & 56 & $\begin{array}{c}73,75 \\
(240 / 23)\end{array}$ & 56 \\
\hline
\end{tabular}

A média geométrica anual de E. coli apresentou-se baixa nas amostras de água bruta provenientes do rio Itajaí-Açu no município de Blumenau, SC, Brasil. Quando realizada a correlação entre a presença dos protozoários e os níveis de coliformes, verificou-se não haver correlação entre ambos. Entretanto, mesmo com valores baixos para o indicador microbiológico, verificou-se a ocorrência dos protozoários no manancial. Segundo Horman et al. (2004) isso pode ser explicado, em partes, devido ao fato de que cada espécie possui um tempo de sobrevivência diferente no meio ambiente, sendo que os protozoários apresentam maior sobrevida do que os indicadores fecais. Para Tallon et al. (2005), mais trabalhos precisam ser feitos para correlacionar a presença de E. coli com a presença de patógenos, a fim de avaliar a necessidade da utilização de indicadores adicionais. Os resultados deste trabalho indicam a necessidade do monitoramento do manancial para presença de Giardia spp. e Cryptosporidium spp. independentemente dos resultados de E. coli na água tratada.

\subsection{Análise pluviométrica}

Os dados pluviométricos indicaram que os meses de janeiro, março e junho de 2014 foram os que apresentaram maior volume registrado ao longo de um mês, com valores acima de 204,9 mm (Figura 2). No mês de junho, o município registrou o maior volume do período com uma precipitação de $331,1 \mathrm{~mm}$, sendo que 116,2 $\mathrm{mm}$ foram registrados em apenas quatro dias. Em relação à precipitação no dia da coleta da água bruta e a ocorrência de protozoários, verificou-se uma correlação positiva fraca para a ocorrência de Giardia $\mathrm{spp}$. $(\mathrm{r}=0,18 ; p=0,54$, $\mathrm{n}=67)$ e Cryptosporidium spp. $(\mathrm{r}=0,36 ; p=0,22 ; \mathrm{n}=67)$. 


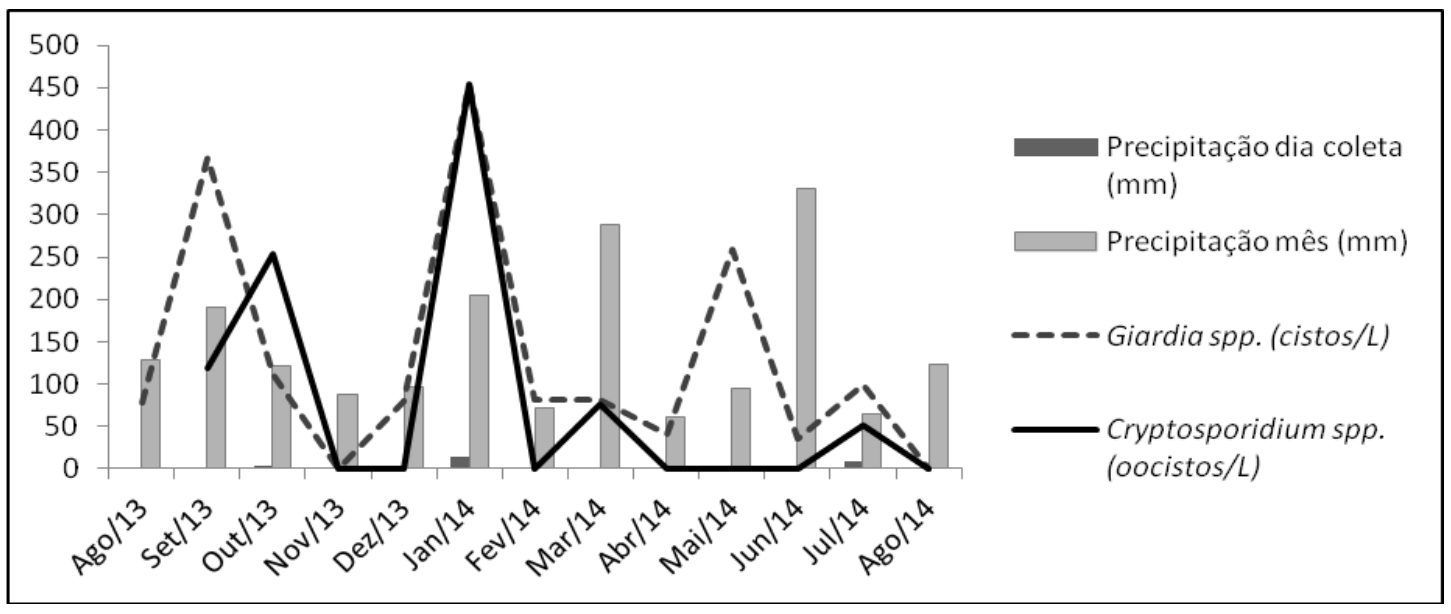

Figura 2. Ocorrência de Giardia spp. e Cryptosporidium spp. nas estações de tratamento de água no município de Blumenau, SC, Brasil e valores de precipitação do dia e mês de coleta da amostra.

Estudos realizados na bacia do Rio Itajaí-Açu demonstram a presença dos protozoários patogênicos Giardia spp. e Cryptosporidium spp. na população. Em pesquisa realizada por Andrade et al. (2008), os autores analisando a prevalência de parasitoses intestinais em crianças entre 0 e 6 anos de idade, verificaram que 18,9\% apresentaram cistos de Giardia spp. e 7,6\%, oocistos de Cryptosporidium spp. em amostras fecais. Volotão et al., (2008) realizaram a genotipagem de Giardia spp. detectada em fezes de bugios ruivos (Alouatta clamitans) mantidos em cativeiro na cidade de Indaial, SC e verificaram a presença da assembleia A1 nas amostras. Este genótipo também infecta seres humanos, indicando que a transmissão de Giardia duodenalis entre humanos e macacos possivelmente ocorra por meio da água contaminada.

A presença de Giardia spp. em praticamente todos os meses de coleta nas amostras do Rio Itajaí-Açu indica que a população, e adicionalmente os animais, podem estar eliminando os protozoários patogênicos pela via fecal contaminando o manancial.

Outra preocupação com a ocorrência de Giardia spp. e Cryptosporidium spp. no Rio Itajaí-Açu consiste no fato de este ser utilizado por pescadores locais e como área de lazer em períodos quentes do ano, favorecendo o contato das pessoas com os protozoários, mediante ingestão acidental da água do rio. E, em extensão, os animais de criação que utilizam as águas do rio para dessedentação podem se contaminar ingerindo as formas infectantes dos protozoários. Segundo Dias et al. (2008), animais domésticos são fontes de contaminação de mananciais por protozoários patogênicos quando os dejetos não são adequadamente tratados e lançados in natura no rio.

\section{CONCLUSÃO E RECOMENDAÇÕES}

Como primeiro registro da ocorrência de Giardia spp. e Cryptosporidium spp. no Rio Itajaí-Açu, reforça-se a importância do monitoramento desses protozoários nos mananciais utilizados para captação de água, levando-se em consideração a vulnerabilidade das barreiras químicas e físicas dos sistemas de tratamento de água em relação à remoção de parasitas. Em vista dos resultados da pesquisa, recomenda-se:

- A coleta e o tratamento adequado dos efluentes produzidos na região evitando que os mesmos atinjam os mananciais utilizados para o abastecimento público;

- Monitoramento constante da presença de protozoários patogênicos nos pontos de captação de água das ETAs do município;

- Melhoria dos sistemas de tratamento de água das ETAs, garantindo avanços nos 
processos de filtração, a fim de manter os valores mínimos de turbidez, conforme exigido pela Portaria 2914/2011 (Brasil, 2011);

- Maior fiscalização por meio do Vigi Água, setor responsável pelo monitoramento da qualidade da água produzida no município;

- Orientação à população, por meio das Unidades de Saúde, da necessidade de se evitar contato com a água do rio, principalmente após períodos de chuva.

\section{AGRADECIMENTOS}

À Prefeitura Municipal de Blumenau por meio do Serviço Autônomo Municipal de Água e Esgoto - SAMAE pelos dados microbiológicos fornecidos aos autores. A Coordenação de Aperfeiçoamento de Pessoal em Nível Superior (CAPES) pela concessão da bolsa de mestrado a primeira autora. A Fundação Universidade Regional de Blumenau (FURB) por meio do Programa de Pós-Graduação em Engenharia Ambiental pelo apoio, orientação e uso das instalações e a Universidade Estadual de Campinas (UNICAMP) pelo treinamento e contribuições no trabalho.

\section{REFERÊNCIA}

ANDRADE, F.; RODE, G.; SILVA FILHO, H. H.; GREINERT-GOULART, J. Parasitoses intestinais em um centro de educação infantil público do município de Blumenau (SC), Brasil, com ênfase em Cryptosporidium spp. e outros protozoários. Revista de Patologia Tropical, v. 37, n. 4, 2008.

AMERICAN PUBLIC HEALTH ASSOCIATION - APHA. Microbiological Examination. In: APHA; AWWA; WEF. Standard methods for the examination of water and wastewater. 21 ed. Washington, 2005.

BASTOS, R. K. X.; HELLER, L.; VIEIRA, M. B. C. M.; BRITO, L. L. A. ; BEVILACQUA, P. D.; NASCIMENTO, L. E. Giardia cysts and Cryptosporidium oocysts dynamics in Southeast Brazil. Occurrence in surface water and removal in water treatment processes. Water Science and Technology, v. 14, p. 15-22, 2004.

BLUMENAU. Plano municipal de saneamento. Blumenau, 2009. Disponível em: www.samae.com.br/arquivos/Plano_de_saneamento.pdf. Acesso em: 05 set. 2014.

BUMENAU. Serviço Autônomo Municipal de Água e Esgoto - SAMAE. ETAs. 2013. Disponível em: http://www.samae.com.br/etas.asp. Acesso em: 21 mar. 2013.

BRASIL. Ministério da Saúde. Portaria n ${ }^{\circ}$ 2914, de 12 de Dezembro de 2011. Diário Oficial [da] União, Brasília, n. 239, 14 de dez. 2011, Seção 1, p. 39.

BRASIL. Ministério do Meio Ambiente; INSTITUTO CHICO MENDES DE CONSERVAÇÃO DA BIODIVERSIDADE. Plano de manejo do parque nacional da Serra do Itajaí. Brasília, 2009.

CANTUSIO NETO, R.; FRANCO, R. M. B. Ocorrência de oocistos de Cryptosporidium spp. e cistos de Giardia spp. em diferentes pontos do processo de tratamento de água, em Campinas, São Paulo, Brasil. Higiene Alimentar, v. 18, p. 52-59, 2004.

CANTUSIO NETO, R.; SANTOS, L. U.; SATO, M. I. Z.; FRANCO, R. M. B. Cryptosporidium spp. and Giardia spp. in samples of natural water from the Atibaia River, city of Campinas, São Paulo, Brazil. Water Science Technology, v. 62, n. 1, p. 217-222, 2010. 
CARVALHO-ALMEIDA, T. T.; PINTO, P. L. S.; QUADROS, C. M. S.; TORRES, M. A. G. V.; KANAMURA, H. Y.; CASIMIRO, A. M. Detection of Cryptosporidium sp. in nondiarrheal faeces from children in a day care center in the city of São Paulo, Brazil. Revista Instituto de Medicina Tropical, v. 48, p. 27-32, 2006. http://dx.doi.org/10.1590/S0036-46652006000100006

CONSELHO NACIONAL DO MEIO AMBIENTE - CONAMA. Resolução n 357/05. Estabelece a classificação das águas doces, salobras e salinas do Território Nacional. Brasília: SEMA, 2005.

CROCKETT, C. S. The significance of streambeds sediments as a reservoir of Cryptosporidium oocysts. 2004. Dissertation (Doctor of Philosophy) - Drexel University Philadelphia, 2004.

DIAS, G. M. F.; BEVILACQUA, P. D.; BASTOS, R. K. X.; OLIVEIRA, A. A.; CAMPOS, G. M. M. Giardia spp. e Cryptosporidium spp. em água de manancial superficial de abastecimento contaminada por dejetos humano e animal. Arquivo Brasileiro Medicina Veterinária e Zootecnia, v. 60, p. 1291-1300, 2008.

FRANCO, R. M. B.; ROCHA-EBERHARDT, R.; CANTUSIO NETO, R. Occurrence of Cryptosporidium oocysts and Giardia cysts in raw water from the Atibaia river, Campinas, Brazil. Revista do Instituto de Medicina Tropical, v. 43, n. 2, p. 109-111, 2001. http://dx.doi.org/10.1590/S0036-46652001000200011

FRANCO, R. M. B.; HACHICH, E. M.; SATO, M. I. Z.; NAVEIRA, R. M. L.; SILVA, E. C.; CAMPOS, M. M. C. et al. Avaliação da performance de metodologias de detecção de Cryptosporidium spp. e Giardia spp. em água destinada ao consumo humano, para o atendimento às demandas da vigilância em saúde ambiental no Brasil. Epidemiologia e Serviços em Saúde, v. 21, p. 233-242, 2012. http://dx.doi.org/10.5123/S167949742012000200006

GONÇALVES, E. M. N.; SILVA, A. J.; EDUARDO, M. B. P.; UEMURA, I. H.; MOURA, I. N. S.; CASTILHO, V. L. P. et al. Multilocus genotyping of Cryptosporidium hominis associated with diarrhea outbreak in a day care unit in São Paulo. Clinics, v. 61, p. 11926, 2006. http://dx.doi.org/10.1590/S1807-59322006000200006

HORMAN, A.; RIMHANEN-FINNE, R.; MAUNULA, L.; VON BONSDORFF, C. H.; TORVELA, N.; HEIKINHEIMO, A.; HANNINEN, L. Campylobacter spp., Cryptosporidium spp., noroviruses, and indicator organisms in surface water in southwestern Finland. Applied and Environmental Microbiology, v. 70, n. 1, p. 95105, 2004. http://dx.doi.org/10.1128/AEM.70.1.87-95.2004

INSTITUTO BRASILEIRO DE GEOGRAFIA E ESTATÍSTICA - IBGE. Cidades. 2010. Disponível em: http://www.ibge.gov.br. Acesso em: 10 mar. 2013.

KARANIS, P.; KOURENTI, C.; SMITH, H. Waterborne transmission of protozoan parasites: A worldwide review of outbreaks and lessons learnt. Journal of Water and Health, v. 5, n. 1, p. 1-38, 2007.

KUMAR, T.; MAJID, M. A. A.; ONICHANDRAN, S.; JATURAS, N.; ANDIAPPAN, H.; SALIBAY, C. C. et al. Presence of Cryptosporidium parvum and Giardia lamblia in water samples from Southeast Asia: towards an integrated water detection system. Infectious Diseases of Poverty, 2016. http://dx.doi.org/10.1186/s40249-016-0095-z 
LOBO, M. L.; XIAO, L.; ANTUNES, F.; MATOS, O. Occurrence of Cryptosporidium and Giardia genotypes and subtypes in raw and treated water in Portugal. Letters in Applied Microbiology, v. 48, n. 6, p. 732-7, 2009. http://dx.doi.org/10.1111/j.1472765X.2009.02605.x

NISHI, L.; VIEIRA, A. M. S.; VIEIRA, M. F.; SILVA, G. F.; BERGAMASCOA, R. Application of hybrid process of coagulation/flocculation and membrane filtration for the removal of protozoan parasites from water. Procedia Engineering, v. 42, p. 148160, 2012. http://dx.doi.org/10.1016/j.proeng.2012.07.405

ODEBRECHT AMBIENTAL. Blumenau. 2016. Disponível em: http://www.odebrechtambiental.com/. Acesso em: 22 fev. 2016.

ROCKWELL, R. L. Giardia lamblia e giardiasis with particular attention to the Sierra Nevada. 19 fev. 2003. Disponível em: http://pweb.jps.net/ prichins/giardia.htm. Acesso em: 10 jan. 2014.

SATO, M. I. Z.; GALVANI, A, T., PADULA, J. A., NARDOCCI, A. C., LOURETTO, M. de S., RAZZOLINI, M. T. P. et al. Assessing the infection risk of Giardia and Cryptosporidium in public drinking water delivered by surface water systems in Sao Paulo State, Brazil. Science of the Total Environment, v. 442, p. 389-396, 2013. http://dx.doi.org/10.1016/j.scitotenv.2012.09.077

SAVIOLI, L.; SMITH, H.; THOMPSON, A. Giardia and Cryptosporidium join the "Neglected Diseases Initiative". Trends in Parasitology, v. 22, p. 203-208, 2006. http://dx.doi.org/10.1016/j.pt.2006.02.015

TALLON, P.; MAGAJNA, B.; LOFRANCO, C.; LEUNG, K. Microbial indicators of faecal contamination in water: a current perspective. Water Air and Soil Pollution, v. 166, p. 1139-1166, 2005.

TRATA BRASIL. Saneamento, nova realidade: o Brasil está muito longe do ideal. 2013. Disponível em: http://www.tratabrasil.org.br/novo_site/?id=5760. Acesso em: 14 abr. 2015.

UNITED STATES. Environmental Protection Agency - USEPA. Method 1623.1: Cryptosporidium and Giardia in water by filtration/IMS/FA. EPA-816-R-12-001. Washington, 2012.

VOLOTÃO, A.; SOUZA JR., J. C.; GRASSINI, C.; PERALTA, J. M.; FERNANDES, O. Genotyping of Giardia duodenalis from Southern brown howler monkeys (Alouatta clamitans) from Brazil. Veterinary Parasitology, v. 158, p. 133-137, 2008. http://dx.doi.org/10.1016/j.vetpar.2008.07.003

WANG, R.; ZHANG, X.; ZHU, H.; ZHANG, L.; FENG, Y.; JIAN, F. et al. Genetic characterizations of Cryptosporidium spp. and Giardia duodenalis in humans Henan, China. Experimental Parasitology, v. 127, p. 42-45, 2011. http://dx.doi.org/10.1016/j.exppara.2010.06.034

WORLD HEALTH ORGANIZATION - WHO. Guidelines for drinking-water quality. 4. ed. Geneva, 2011. 